\title{
Analysis of Ship RCS Detected by Multifrequency HFGWR
}

\author{
Ke Sun, Biyang Wen, and Ruokun Wang \\ Radar and Signal Processing Laboratory, Electronic Information School, Wuhan University, Wuhan 430072, China \\ Correspondence should be addressed to Biyang Wen; bywen@whu.edu.cn
}

Received 22 January 2017; Revised 28 March 2017; Accepted 2 May 2017; Published 18 May 2017

Academic Editor: Pierfrancesco Lombardo

Copyright (C) 2017 Ke Sun et al. This is an open access article distributed under the Creative Commons Attribution License, which permits unrestricted use, distribution, and reproduction in any medium, provided the original work is properly cited.

\begin{abstract}
One of the important applications of high frequency-ground wave radar (HFGWR) is to detect offshore ships. A proper method should be used to obtain the ship radar cross section (RCS), which is a key parameter of the ship. This paper proposes a method based on an automatic information system (AIS). The relationship of the ship RCS versus bearing for different frequencies is analyzed by processing multifrequency HFGWR data. With this new method, bearing information is taken into consideration, which is not the case in traditional empirical formulas. The results provide prior knowledge for ship detection and tracking; therefore, the probability of detection is significantly improved.
\end{abstract}

\section{Introduction}

Over-the-horizon radar, HF ground-wave radar (HFGWR), has emerged in the past few decades for both monitoring ocean kinetic parameters (e.g., current, wave, and wind) and detecting moving targets on the ocean surface [1-5]. Such systems are relatively inexpensive and convenient. Vertically polarized $\mathrm{HF}$ (3-30 MHz) electromagnetic (EM) waves can propagate over the horizon along the sea surface with low loss and resonate with targets at sea. HFGWR provides overthe-horizon, all-weather, continuous, and real-time detection of a large sea area. The radar cross section (RCS) provides the ability to scatter EM waves transmitted by radar, which is fundamental for target recognition and detection. Targets are generally confined to ships offshore. In field experiments, targets from tens of meters to a hundred meters in size can be detected in the resonance region of the EM waves in the HF band. Small targets can be detected more easily at the upper end of the HF band. However, we need to detect targets that will cover a range of bearings and can be tracked for a long time. Therefore, relatively large targets are of interest in this study.

There is no simple formula applicable for calculating the RCS in the resonance region. The RCS is typically obtained by numerical simulation and experimental measurements. However, due to experimental conditions and cost constraints, it is challenging for HFGWR to obtain a large number of ship RCSs with experimental measurements. Certain numerical simulation methods, for example, the method of moments $(\mathrm{MOM})$ and the finite difference time domain method (FDTD), have been applied to analyze RCSs in the HF bands [6, 7]. Detailed ship information (e.g., structure, material, and size) is required to simplify the ship model. The effect of a rough ocean surface, randomly fluctuating waves, and the interaction of nearby ships should also be taken into consideration. Therefore, these numerical simulation methods are relatively cumbersome [8]. Researchers in related fields have extracted empirical formulas by analyzing a large amount of data. For example, (1) has primarily been used to calculate the RCS in X, S, and L band radar applications [9], and Ponsford applied it to HFGWR [10].

$$
\mathrm{RCS}=52 f^{1 / 2} D^{3 / 2},
$$

where $f$ is the operating frequency of the HFGWR (in MHz) and $D$ is the full displacement (in kilotons). This equation is a static description of the RCS, which is a constant for a certain ship when $f$ is stable.

By analyzing experimental data of HFGWR, Barrick proposed that the target echo is proportional to the sixth power of the height of the mast [11], which remained consistent with the static characteristic of the RCS. The above studies provide rough descriptions of the RCS and fit experimental measurements obtained from specific situations. However, these methods do not address the effect of bearing on the RCS, which is critical in real radar applications. For example, 
ship echoes may disappear suddenly when the ship changes course.

Because of the limitations of previous RCS studies, a more convenient and comprehensive method should be developed. This paper proposes a method based on experimental HFGWR data with the help of automatic information system (AIS) information. A large number of targets are analyzed, and the relationship of the RCS versus the bearing for a variety of frequencies is obtained.

\section{Method of Data Processing}

The radar equation for HFGWR is

$$
P_{r}=\frac{P_{t} G_{t} G_{r} \sigma F^{4} \lambda^{2}}{(4 \pi)^{3} R^{4} L_{s}},
$$

where $R$ is the range of the target, $P_{t}$ is the average transmission power, $P_{r}$ is the receiving power, $G_{t}$ is the transmitting antenna gain, $G_{r}$ is the receiving antenna gain, $\sigma$ is the RCS, $F$ is the Norton attenuation factor, $\lambda$ is the EM wavelength, and $L_{s}$ is the system attenuation factor [12].

When the HFGWR operates in steady state, $P_{t}, G_{t}, G_{r}$, and $L_{s}$ can be considered constant. Equation (2) can be expressed as

$$
\sigma=C \cdot \frac{R^{4}}{F^{4} \lambda^{2}} \cdot P_{r}
$$

where $C=(4 \pi)^{3} L_{s} / P_{t} G_{t} G_{r}$ is a constant for a particular radar deployment. $\lambda$ is also an invariant when the radar frequency is stable. Thus, $\sigma$ can be expressed as

$$
\sigma \propto \frac{R^{4}}{F^{4}} \cdot P_{r} .
$$

The attenuation factor $F$ is related to the frequency, polarization, propagation path, and scattering of rough ocean surfaces, among other factors. Barrick calculated the influence of a rough ocean surface on EM wave propagation attenuation based on EM field theory [13, 14]. The data used in this paper were collected over a short time when the HFGWR had been operating steadily and the sea state changed minimally in beam width. In this case, other factors have minimal influences on $F$ except for the distance of EM wave propagation.

Both the echo power of ships and Bragg waves decline when distance increases. In the case of a fully developed sea surface, Barrick proposed that the RCS of Bragg wave echo per unit area is invariant [15] that can be expressed as

$$
\sigma_{0}=0.02=-17 \mathrm{~dB} \text {. }
$$

Based on this equation, the RCS of the Bragg wave echo at different ranges is invariant. From (3), the power differences of the Bragg wave echo at different ranges are caused by $R$ and $F$. Then, the rule of $F$ can be obtained. $F$ fluctuates significantly when processing different HFGWR data. In actual experimental conditions, the assumption of a fully developed ocean is no longer established; therefore, $F$

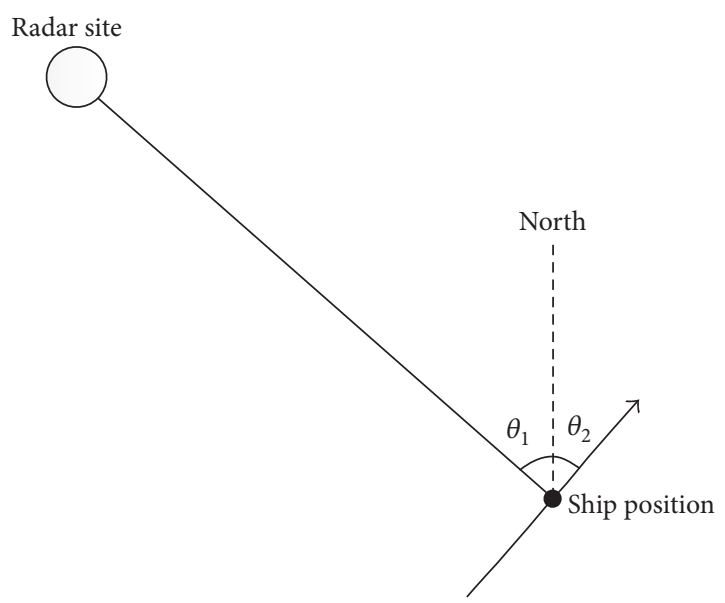

FIGURE 1: Bearing of ship to radar site.

may fluctuate. An analysis of HFGWR data shows that the difference in echo power is small for one target at a certain bearing in close distance (no more than 2 range bins). In this case, $F$ can be considered an invariant. The following studies are based on this type of target, which is defined as a matched target.

The AIS information provides characteristics of offshore ships including location, velocity, and heading. By matching AIS information and HFGWR data, some matched targets can be obtained. The bearings to the radar site of these targets can be calculated with the location of the radar site, the targets, and the heading of the targets (defining the bearing to be $0^{\circ}$ when the bow is facing the radar site and $180^{\circ}$ when the stern is facing the radar site), as illustrated in Figure 1. The arrow represents the heading of this ship. $\theta_{1}$ is calculated from the locations of the radar site and the ship. $\theta_{1}$ represents the heading of the ship. The bearing to the radar site of this ship is $\theta=\theta_{1}+\theta_{2}$. This value changes when the ship is positioned at different locations or has a different heading.

The HFGWR used in this paper is a wide beam radar. Therefore, a digital beamforming (DBF) algorithm should be implemented before calculating the power of target echoes. When the receiving antenna is determined, the DBF weighting values at different bearings are identical; thus, there is no need to consider the problem of weight compensation at different bearings.

As described above, the RCS of the matched targets can be expressed as

$$
\sigma(\theta) \propto R^{4} \cdot P_{r}(\theta)
$$

Although the absolute value of RCS is not obtained, the relationship of RCS versus bearing can be characterized using the target power. To illustrate this relationship more clearly, it is normalized as (7) in the following process:

$$
\sigma_{\text {norm }}(\theta)=\frac{\sigma(\theta)}{\max \langle\sigma(\theta)\rangle},
$$

where $\max \langle\sigma(\theta)\rangle$ represents the maximum of $\sigma(\theta)$. 


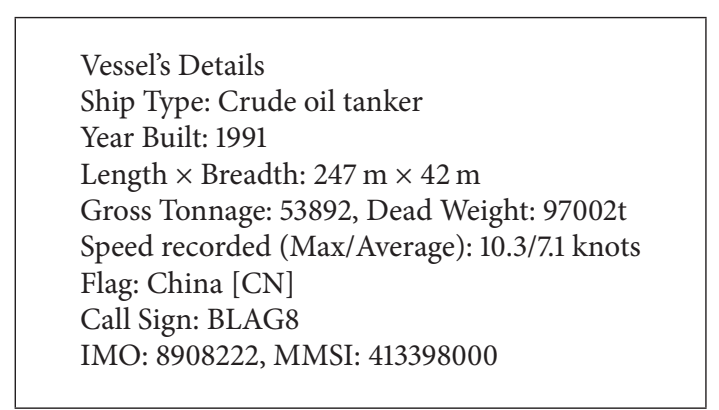

Box 1: AIS information of one matched target.

\section{Description of Experimental System and Radar Data Processing}

The experimental data used in this paper is collected with a multifrequency HFGWR system. This system operates at several different carrier frequencies by time-division multiplexing, and its transmitted waveform is a linear frequencymodulated interrupted continuous wave (FMICW). The sweep bandwidth can be preset so that the distance resolution is adjustable from $1 \mathrm{~km}$ to $5 \mathrm{~km}$. The radar operates at two frequencies, frequency $1(10.8 \mathrm{MHz})$ and frequency 2 $(8.2 \mathrm{MHz})$. The average transmission power is $300 \mathrm{~W}$, and the farthest detection range designed is $150 \mathrm{~km}$. The transmitting antenna is a 3-element Yagi antenna. The receiving antenna is a linear array antenna of eight $2.5 \mathrm{~m}$ passive monopole helical antennas. The antenna system used in the experiment has a wide beam width of $35^{\circ}$ (corresponding to operating frequency $8.2 \mathrm{MHz}$ ) and $24^{\circ}$ (corresponding to $10.8 \mathrm{MHz}$ ). The bearing of the target is calculated by the super resolution algorithm multiple signal classification (MUSIC). The coherent integration time of the HFGWR is sufficiently long that the velocity resolution is relatively high. The targets can be distinguished by their bearing, distance, and velocity differences.

During the field experiment, a set of AIS receivers was used to collect ship information at sea (within approximately $60 \mathrm{~km}$ ), including location, heading, and velocity. The AIS information can be used as auxiliary information for signal processing [16].

The following results were obtained from HFGWR field experimental data collected at the Zhujiajian radar site (122.4275 E, 29.8931 N), Zhejiang Province, China, on August 29, 2010. After detecting and matching, the relationship of the RCS versus the bearing can be obtained from the echo power of the ships and the bearing to the radar site.

Figure 2 shows the results of one radar data set; the green line represents AIS information, and the black dot lines are ship tracks extracted from the radar data. AIS information provides the maritime mobile service identify (MMSI) of ships; the appearance information (e.g., size and tonnage) of a ship can be obtained by searching its MMSI on the Internet, as shown in Box 1.

The corresponding dynamic information, such as location, heading, and velocity, can be received and decoded by the AIS receiver.

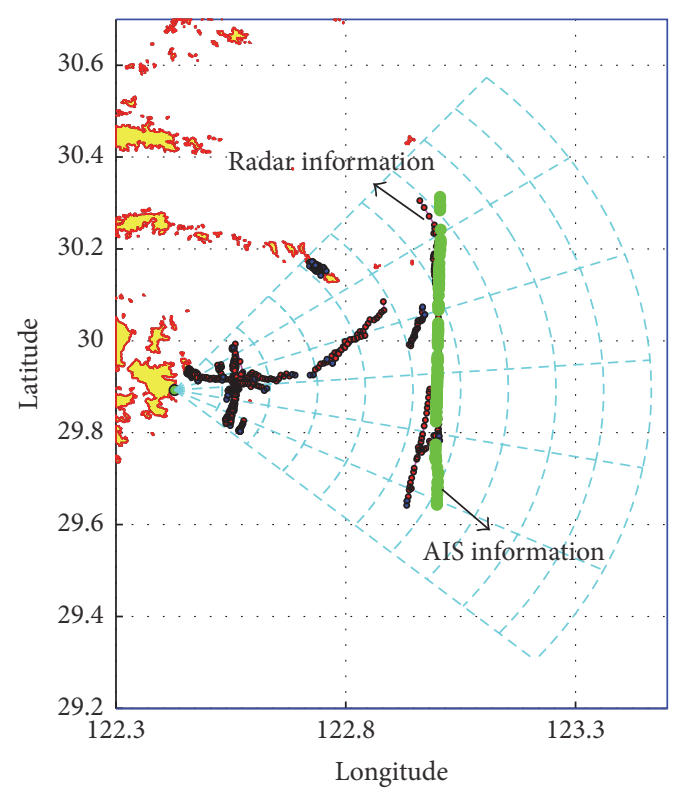

FIGURE 2: Results of target detection and matched AIS information.

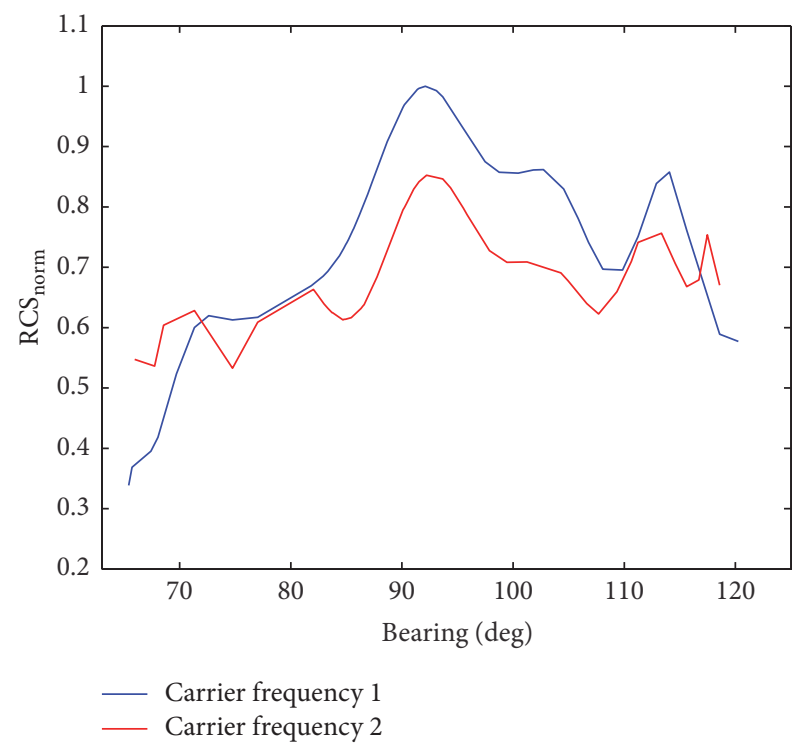

FIGURE 3: RCS versus bearing at two frequencies for one target.

Figure 3 shows the relationship of the RCS versus bearing for two frequencies. The RCSs are normalized to their maximum values at frequency 1 . The changing trends of the RCS for both frequencies are almost the same, but at certain bearings, their trends are not consistent. This observation illustrates that multifrequency HFGWR is better for target detection than single-frequency HFGWR, particularly for the case in which a target disappears at one frequency. The theoretical RCS ratio for one target of the two frequencies is 0.8714 as calculated by (1). The RCS ratio in Figure 3 is calculated and shown in Figure 4 as a solid line, and the theoretical values are shown as a dotted line. Some fluctuating 


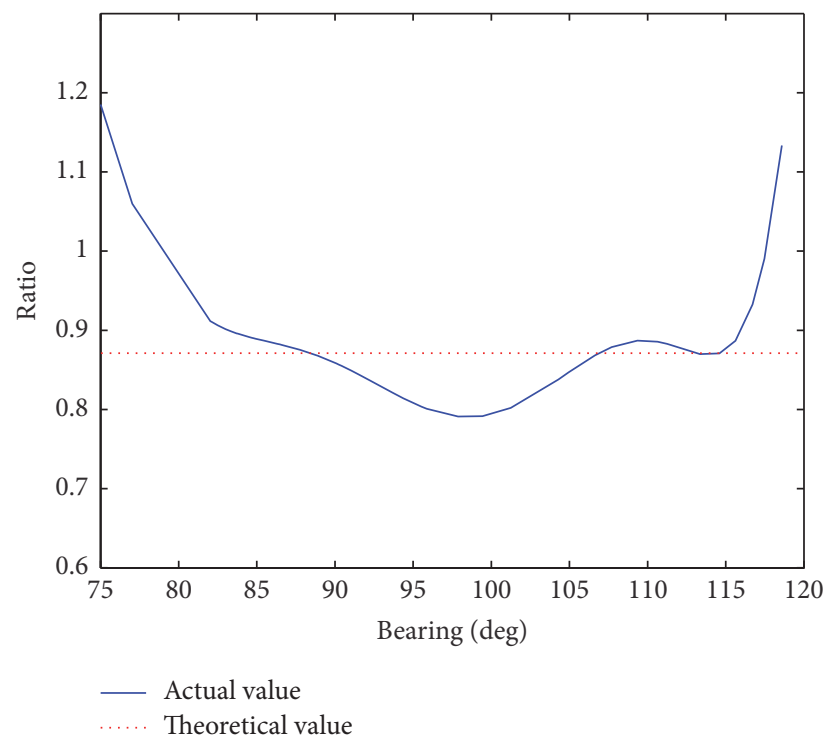

FIGURE 4: RCS ratio of two frequencies for one target.

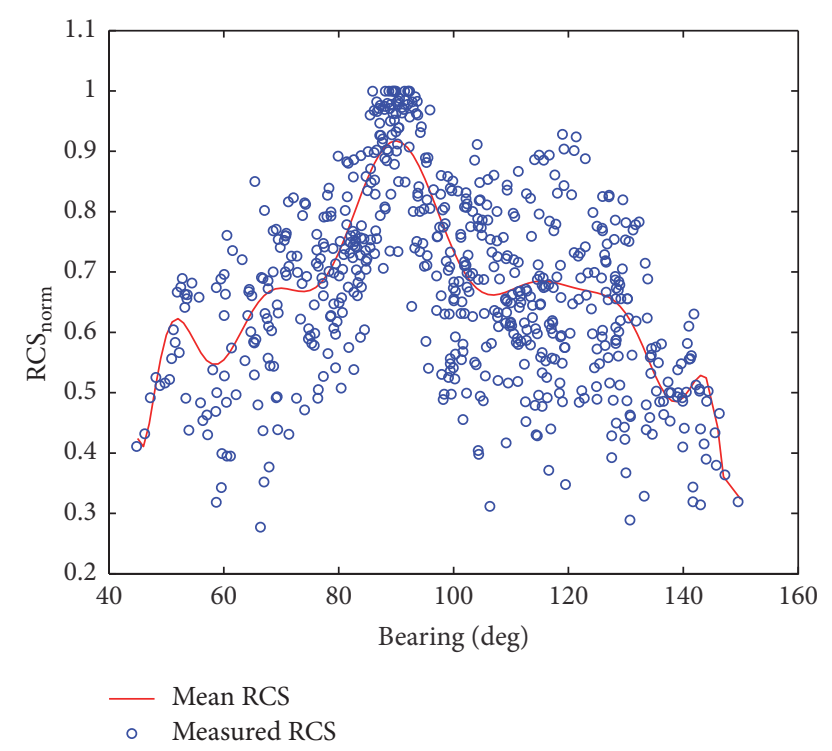

FIGURE 5: RCS versus bearing for 15 targets.

values on the edge are excluded. Two lines match well, which verifies the feasibility of the method in this paper.

To obtain the overall characteristics of ship RCSs, the influence of the ship size on the RCS must be decoupled and eliminated. Fifteen matched ships with similar sizes are chosen, all approximately $250 \mathrm{~m}$ in length. The bearings of these ships range from $40^{\circ}$ to $160^{\circ}$. Other ships are beyond the detection range or have an undetectably low RCS.

The RCSs of these ships are calculated by the proposed method above (for frequency 1). For each ship and bearing, the RCS is plotted as blue circles in Figure 5. The red line represents the RCS versus bearing after averaging and smoothing the values denoted by the blue circles. A significant fluctuation of the RCS versus bearing is observed. Due to lack of bearing information, the traditional empirical formulas are insufficiently comprehensive to describe the RCS characteristics. The peak of the RCS appears in the $90^{\circ}$ direction, which indicates that the ships present the largest scattering area when positioned broadside.

\section{Conclusion}

This paper proposed a method to obtain the relationship of the RCS versus bearing based on HFGWR experimental data. The result is similar to certain numerical simulations $[17,18]$, but the method in this paper is simpler and more convenient; a large number of ships are studied. The RCS ratio at two frequencies is consistent with the theoretical value, which verifies the method presented in this paper. Information on bearing is taken into consideration, in contrast to traditional methods. The results provide a reference in designing target detection and tracking contrast experiments and target recognition. Target RCS estimated in this paper can provide more comprehensive information of targets, which is helpful for selecting the proper detection method and threshold. Therefore, the target detection probability is improved to some extent.

\section{Conflicts of Interest}

The authors declare that there are no conflicts of interest regarding the publication of this paper.

\section{Acknowledgments}

This work was supported by the National Natural Science Foundation of China (61671331) and the National Key Scientific Instruments and Equipment Development Project of China (2013YQ160793).

\section{References}

[1] Y. Lai, H. Zhou, and B. Wen, "Surface current characteristics in the taiwan strait observed by high-frequency radars," IEEE Journal of Oceanic Engineering, 2016.

[2] H. Zhou and B. Wen, "Wave height extraction from the firstorder bragg peaks in high-frequency radars," IEEE Geoscience and Remote Sensing Letters, vol. 12, no. 11, pp. 2296-2300, 2015.

[3] J. Yang, R. Wang, Y. Shi et al., "Dual-use multistatic HF ocean radar for current mapping and ship tracking," IEICE Electronics Express, vol. 11, no. 12, 2014.

[4] H. J. Roarty, E. R. Lemus, E. Handel, S. M. Glenn, D. E. Barrick, and J. Isaacson, "Performance evaluation of seasonde highfrequency radar for vessel detection," Marine Technology Society Journal, vol. 45, no. 3, pp. 14-24, 2011.

[5] A. M. Ponsford and J. Wang, "A review of high frequency surface wave radar for detection and tracking of ships," Turkish Journal of Electrical Engineering \& Computer Sciences, vol. 18, no. 3, pp. 409-428, 2010.

[6] G. Cakir and L. Sevgi, "Radar cross-section (rcs) analysis of high frequency surface wave radar targets," Turkish Journal of Electrical Engineering and Computer Sciences, vol. 18, no. 3, pp. 457-467, 2010.

[7] S. K. Podilchak, "Monostatic and bistatic hf radar cross section analysis of large vessels using feko," Report DRDC OTTAWA CR 2010-262, Defence R\&D, Ottawa, Canada, April 2011. 
[8] J. R. Barnum, "Ship detection with high-resolution HF skywave radar," IEEE Journal of Oceanic Engineering, vol. 11, no. 2, pp. 196-209, 1986.

[9] M. I. Skolnik, "An empirical formula for the radar cross section of ships at grazing incidence," IEEE Transactions on Aerospace and Electronic Systems, vol. 10, article 292, no. 2, 1974.

[10] A. M. Ponsford, "Surveillance of the 200 nautical mile Exclusive Economic Zone (EEZ) using High Frequency Surface Wave Radar (HFSWR)," Canadian Journal of Remote Sensing, vol. 27, no. 4, pp. 354-360, 2001.

[11] D. Barrick and L. Pederson, "Hf surface-wave radar: revisiting a solution for EEZ ship surveillance," EEZ International, pp. 3537, 2004.

[12] R. H. Khan and D. K. Mitchell, "Waveform analysis for highfrequency fmicw radar," IEE Proceedings of Radar and Signal Processing, vol. 138, no. 5, pp. 411-419, 1991.

[13] D. E. Barrick, "Theory of HF and VHF propagation across the rough sea, 1, the effective surface impedance for a slightly rough highly conducting medium at grazing incidence," Radio Science, vol. 6, no. 5, pp. 517-526, 1971.

[14] D. E. Barrick, "Theory of HF and VHF propagation across the rough sea, 2, application to HF and VHF propagation above the sea," Radio Science, vol. 6, no. 5, pp. 527-533, 1971.

[15] D. E. Barrick, "First-order theory and analysis of MF/HF/VHF scatter from the sea," IEEE Transactions on Antennas and Propagation, vol. 20, no. 1, pp. 2-10, 1972.

[16] X. Huang, B. Wen, and F. Ding, "Ship detection and tracking using multi-frequency HFSWR," IEICE Electronics Express, vol. 7, no. 6, pp. 410-415, 2010.

[17] S. K. Podilchak, H. Leong, R. Solomon, and Y. M. M. Antar, "Radar cross-section modeling of marine vessels in practical oceanic environments for high-frequency surface-wave radar," in Proceedings of 2009 IEEE Radar Conference, RADAR 2009, pp. 1-6, May 2009.

[18] H. Wilson and H. Leong, "An estimation and verification of vessel radar-cross-sections for HF Surface Wave Radar," in Proceedings of International Conference on Radar, RADAR 2003, pp. 711-716, September 2003. 


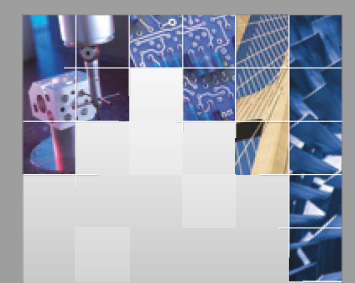

\section{Enfincering}
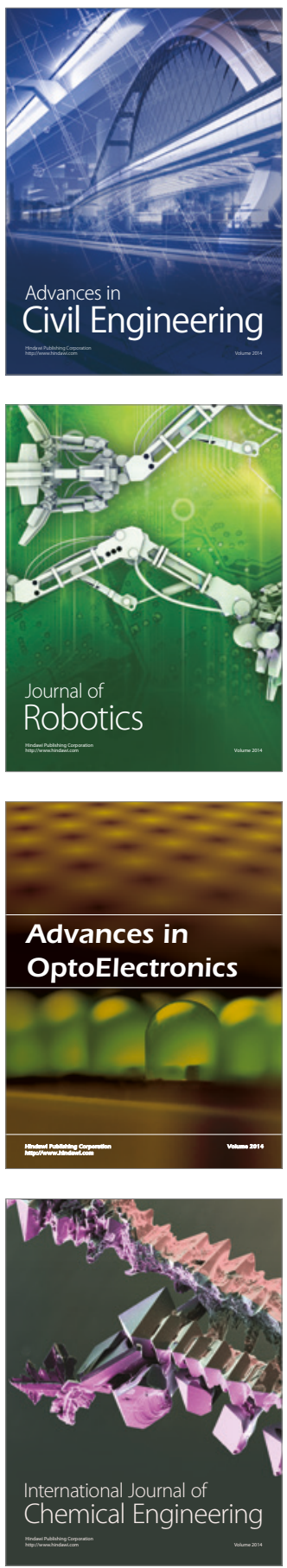

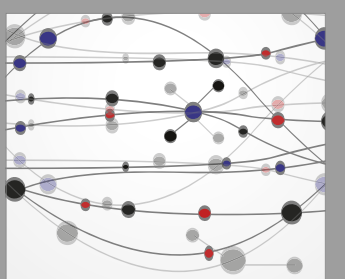

The Scientific World Journal

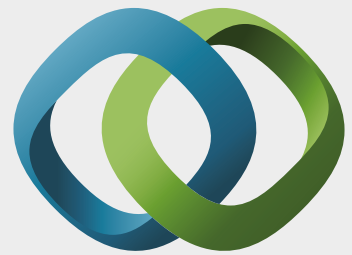

\section{Hindawi}

Submit your manuscripts at

https://www.hindawi.com
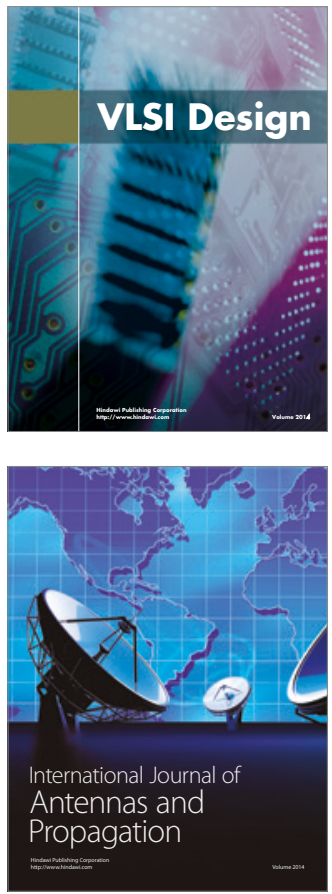

\section{Rotating}

Machinery
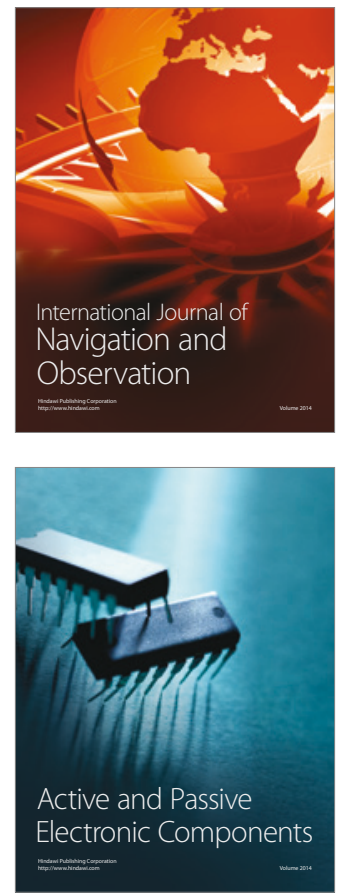
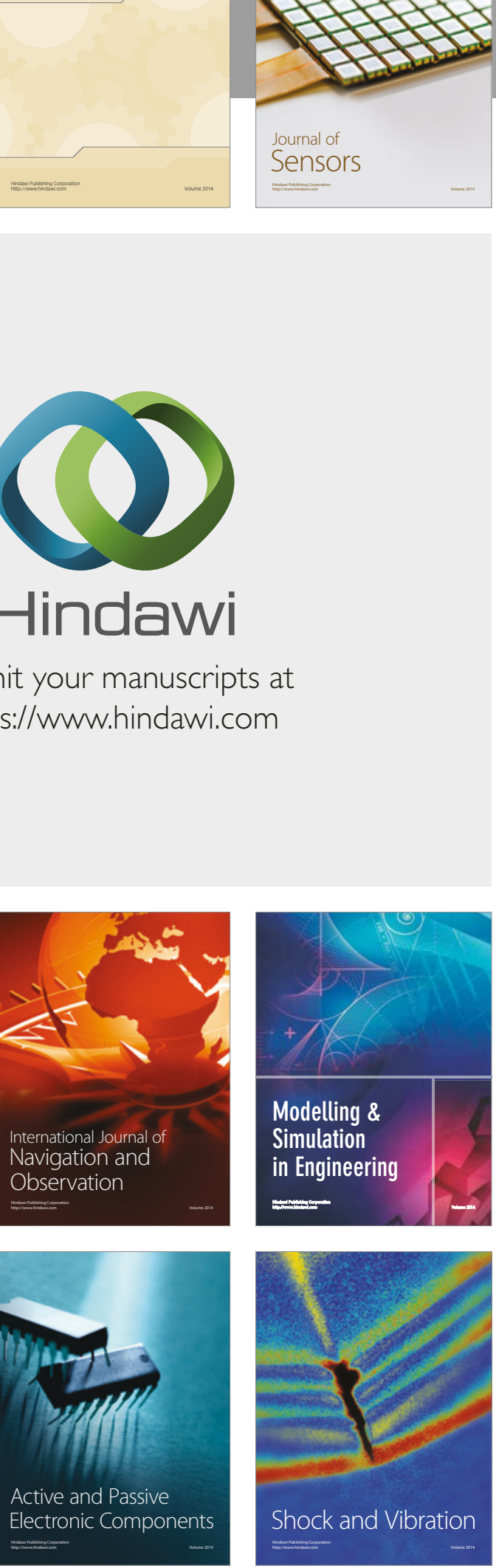
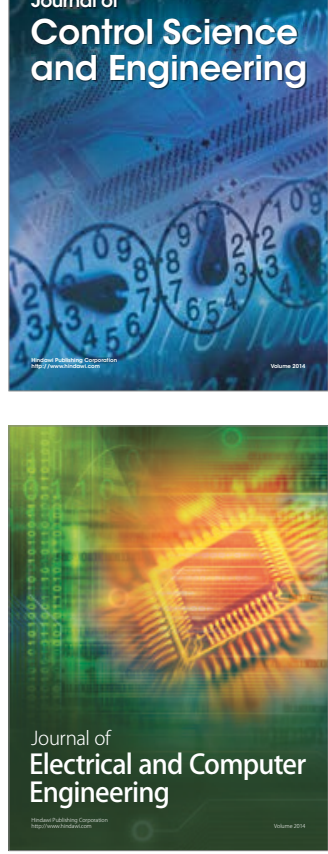

Distributed

Journal of

Control Science

and Engineering
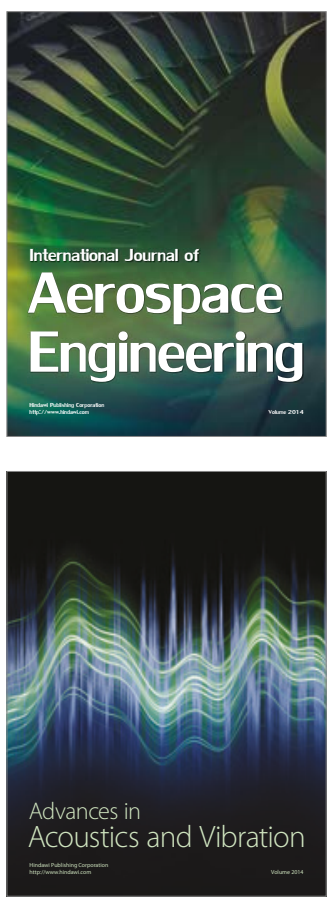

Sensor Networks 